arm seemed to increase in both strength and bone content. As explained in our results, several volunteers admitted exercising both arms. The correlation between grip strength and bone mineral content, however, remained across all groups and at all stages in both the volunteer and fracture studies.

The small loss between the values at the start and those after six months reflects the natural rate of bone mineral loss seen in aging women. Interestingly, there was a corresponding loss in grip strength.

We originally designed an experiment that was to run for a six week period of exercise. We chose to use the raw bone mineral content scores precisely because of the variation in bone mineral content measurements that subtract a supposed circumferential fat layer. They derive rather than measure fat content. In the region examined it is unreasonable to expect a net gain or loss in body fat in a mere six weeks of occasionally squeezing a tennis ball. The relatively large changes in grip strength and the associated significant gains in bone mineral content would in fact outweigh any changes in fat content. We used a routine technique and daily phantom standardisation in our experiment. This reduces the variation in the subject's grip technique and positioning errors, which are probably the greatest source of error in other series.

We agree with Dr Stevenson and Ms Lee's last point: further work is certainly needed on the role of brief periods of skeletal stress in the reversal of osteoporosis. This free and physiological augmentation of the skeleton may, however, be more effective than some current expensive and unproved remedies.

Richmond,

M C BEVERLY

Surrey TW9 1AW

Northampton General Hospita

T A RIDER

Northampton NN1 5BD

Hammersmith Hospital,

M J EVANS

London W12 0HS

Nuffield Orthopaedic Centre,

Oxford OX3 7LD

\section{Cricoid pressure during cardiopulmonary resuscitation}

SIR, - We would like to comment on one aspect in the excellent article by $\mathrm{Mr}$ A K Marsden': the use of cricoid pressure (Sellick's manoeuvre) during two rescuer cardiopulmonary resuscitation by health care professionals.

This procedure requires the operator to use both hands. ${ }^{2}$ It is often used during induction of anaesthesia to prevent pulmonary contamination by gastric contents. Once applied, cricoid pressure must not be relaxed until the trachea is protected by a cuffed endotracheal tube. Any period without cricoid pressure allows passive regurgitation into the pharynx; hence pulmonary soiling is possible with positive pressure ventilation, rendering the technique pointless. During two rescuer cardiopulmonary resuscitation it is not possible for one person to apply and maintain cricoid pressure continuously while performing chest compression. Thus we believe that a third person must be available to apply cricoid pressure, as recommended in the American standards for cardiopulmonary resuscitation.

Training of health care professionals in this manoeuvre must be thorough because poorly applied cricoid pressure distorts the anatomy, making ventilation and intubation more difficult. To our knowledge no suitable training manikin exists to teach this manoeuvre. Adequate training in the application of cricoid pressure can be given in anaesthetic rooms. We believe that this should be encouraged: it would give trainees opportunities to practise airway management skills under experienced supervision in a safe and controlled environment.

N W PENFOLD

M B SMITH

Department of Anaesthesia

Addenbrooke's Hospital,

Cambridge CB2 $2 \mathrm{OQ}$

1 Marsden AK. Guidelines for cardiopulmonary resuscitation. Basic life support. Br Med f 1989;299:442-5. (12 August.)

2 Sellick BA. Cricoid pressure to control regurgitation of stomach contents during the induction of anaesthesia. Lancet 1961;ii: 404-6.

3 Standards and guidelines for cardiopulmonary resuscitation and emergency cardiac care. FAMA 1986;255:2905-84.

4 Churchill-Davidson HC, ed. A practice of anaesthesia. th ed London: W B Saunders, 1979:1364.

\section{Advanced life support}

SIR, - A serious flaw in Dr D A Chamberlain's article on the updated guidelines for treating different modes of cardiac arrest ${ }^{\prime}$ is the absence of any mention of hypoxic cardiac arrest, which is undeniably the cause of death in 1500 asthmatic patients every year in Britain.

It is known from animal experiments and from observing brain dead patients disconnected from ventilators that the most common course of cardiac events in profound hypoxia, such as occurs in severe acute asthma, is not the sudden onset of ventricular fibrillation but progressive sinus bradycardia followed by slow idioventricular rhythm and cardiac arrest in asystole. A vital measure in resuscitating a dying asthmatic patien is therefore to correct hypoxia by immediate intubation and ventilation with $100 \%$ oxygen. If the patient has not progressed beyond the stage of sinus bradycardia or if idioventricular rhythm has only recently supervened this measure will usually return the patient to sinus tachycardia and restore an adequate circulation. Even if cardiac arrest has occurred a sharp blow on the sternum will often restart cardiac action, provided the arrest has been of short duration and the lungs are being adequately ventilated with oxygen. If, however the treatment of such patients does not include reoxygenation and is restricted to the measures shown on the apparent asystole algorithm a fata outcome will be inevitable.

Aside from the special and crucial need for re oxygenation in treating cardiac arrest in asthma and in other conditions causing profound hypoxia, it is strange that the guidelines for prolonged resuscitation and post resuscitation care contain no mention of giving oxygen. Could the reason for that omission, and for the apparent disregard of the problem of hypoxic cardiac arrest, be that the guidelines are mainly orientated towards cardiac arrest in ischaemic heart disease and place insufficient emphasis on conditions in which severe hypoxia is either the primary cause of cardiac arrest or a major contributory factor?

IAN W B GRANT

Balnaclash

Burnwynd

Kirknewton,

West Lothian EH27 8EA

1 Chamberlain DA. Advanced life support. Br. Med J 1989;299. 446-8. (12 August.)

AUTHOR'S REPLY, - The importance of hypoxia as a cause of asystolic cardiac arrest cannot be overemphasised, and Dr Grant's points are well taken. No member of the Resuscitation Council would disagree with them

There is, however, an element of misunderstanding, which I am glad to clarify. My remit for the article on advance life support ' was to provide an apologia for the changes from the 1984 guidelines; this is stated clearly in the introduction. The article was not intended to be a definitive account of advanced life support. The recommended procedures will be discussed more fully in the revised edition of the $A B C$ of Resuscitation, due to be published in the autumn. The algorithms that were published in the recent article came from the new cardiopulmonary resuscitation poster published by the Resuscitation Council. The full poster has adequate emphasis on the importance of the airway, the need for airway adjuncts, the value of oxygen treatment, and the possible need for intubation. These were not included in the algorithms in the article because there were no major changes since 1984. Copies of the full poster may be obtained from the Resuscitation Council, c/o Department of Anaesthetics, Royal Postgraduate Medical School, Hammersmith Hospital, Du Cane Road, London W12 0HF

DOUGLAS CHAMBERIAIN for Resuscitation Council of the United Kingdom Roval Sussex County Hospital, Brighton $\mathrm{BN} 25 \mathrm{BF}$

1 Chamberlain DA. Advanced life support. Br Med J 1989;299: 4+6-8. 12 August.

\section{Ethics of clinical research}

SIR, - The article by Professor Michael Baum and colleagues fails to address some of the more worrying questions provoked by the 1980 Cancer Research Campaign adjuvant breast cancer trial. Several leading international authorities in the drug treatment of cancer were concerned about the ethics of the study before it started. Based on evidence available then, the cyclophosphamide only arm was considered inadequate treatment compared with a three drug combination of cyclophosphamide, methotrexate, and fluorouracil. They were concerned that suboptimal treatment was being given to uninformed patients. These criticisms were originally published in the $B M \mathcal{F}^{2}$ and cited again more recently in the Institute of Medical Ethics Bulletin. ${ }^{*}$

One justification for continuing the trial despite these concerns was that the ethics committee at King's College Hospital had decided that to proceed was ethically proper. As a committee can make judgments only on the evidence presented to it, this raises the question of what the committee members were told. For example, when they decided that informed consent was no longer necessary were they aware of the reservations about the ethics of the trial published in the $B M \mathcal{F}$ or that cyclophosphamide alone would probably be of no benefit to $90 \%$ of the patients receiving it?

The implication that a requirement for informed consent might make patients reluctant to enter a randomised study is only a small part of the problem. Other reasons why patients are not entered into clinical trials generally include reluctance of surgeons and radiotherapists to inform patients that the option exists, inability to meet the trial protocol because of the lack of local facilities, and patients' insistence on adjuvant chemotherapy outside the setting of a trial. Even so, the original good news from Milan that mortality in high risk premenopausal patients with breast cancer can be significantly reduced with cyclophosphamide, methotrexate, and fluorouracil chemotherapy without unacceptable side effects has been confirmed by randomised studies conducted mainly in the United States in which informed consent was obtained." 11

In my opinion most of the ethical controversy raised by the Cancer Research Campaign trial can be reduced to one simple question: Are patients entitled to know what treatment their doctors are giving them and why? I think the answer must always be "Yes." If the King's ethical committee was aware of the trial criticisms it seems to have decided that the answer to this question was "No." 
Whatever genuine differences there may be within the medical profession about the treatment of breast cancer I think that all women who develop this disease have the right to be fully informed, without prejudice, of all of the treatment options that are available.

L A PRICE

United Kingdom Representative,

International Advisory Council,

New York Chemotherapy Foundation.

London WIN IDG

1 Baum M, Zilkha K, Houghton J. Ethics of clinical research: lessons for the future. Br Med f 1989:299:251-3. (22 July.)

Price LA. Breast cancer trials - a new initiative. $B r$ Med $1980 ; 281: 1422$

3 Hill BT. Breast cancer trials-a new initiative. $\mathrm{Br} M e d f$ $1980 \cdot 281: 1565$

4 Spittle M. Breast cancer trials - a new initiative. $\mathrm{Br}$ Med $\mathcal{F}$ 1981;282:68.

5 Holland J. Breast cancer trials-a new initiative, Br Med 7 $1981 ; 282: 221$.

6 Livingston RB. Breast cancer trials-a new initiative. Br Med $\mathcal{F}$ 1981;282:563.

7 Price LA, Hill BT. Breast cancer trials-a new initiative. BrMed F 1981;282:563.

8 Price LA. Chemotherapy options cause ethical problems. Institute of Medical Ethics Bulletin 1988;44:21-2.

9 Glick JH. Meeting highlights: adjuvant therapy for breast cancer. fNCI 1980;80:471-5.

10 Henderson IC. Adjuvant therapy for breast cancer. $N$ Engl $f$ Med 1988;318:434-4

11 Early Breast Cancer Trialists Collaborative Group. Effects of adjuvant tamoxifen and cytotoxic therapy on mortality in early breast cancer: an overview of 61 randomised trials among 28896 women. $N$ Engl f M Med 1988;319:1681-92.

AUTHORS' REPLY, - Dr Price is correct in stating that controversy surrounded the launch of the Cancer Research Campaign adjuvant breast cancer trial as he was one of the leading proponents. $\mathrm{He}$ does not, however, state in his letter that his points were fully addressed at the time ${ }^{1}$ and that, at least on this side of the Atlantic, the majority of patients being treated for early breast cancer did not receive any adjuvant systemic therapy. ${ }^{2}$ Indeed, in 1980 the viewpoint of the majority was that adjuvant chemotherapy, particularly cyclophosphamide, methotrexate, and fluorouracil, probably did more harm than good. ${ }^{3}$ It has subsequently been shown by the world overview of adjuvant therapy in early breast cancer ${ }^{+}$that polychemotherapy is of little benefit in women over the age of 50, who comprised two thirds of the Cancer Research Campaign trial. It can also be argued that cyclophosphamide alone is valuable to only a small proportion of those who receive it, but at least the advantage has been shown in all subgroups in an overview of the Cancer Research Campaign and original Nissen-Meyer trial and the toxicity is mild. 'Treatment with cyclophosphamide, methotrexate, and fluorouracil is also beneficial to only a small proportion of the patients who receive it and yet all have to experience the unpleasant side effects. Judging by recruitment ( 2240 patients in five years), the Cancer Research Campaign trial was certainly not considered unethical by the vast majority of British clinicians, and it must be borne in mind that we only now have the evidence on systemic therapy from the world overview because many patients were randomised into controlled trials.

The ethical committee at King's did receive a full protocol summarising the arguments for and against systemic therapy, but it was evidence of the severe emotional difficulties patients were experiencing in learning of the uncertainty of treatment that made the committee waive the otherwise universal requirement of informed consent. Despite Dr Price's viewpoint the issue of informing patients is not cut and dried. To quote a recent editorial: "To inform someone about clinical disagreement when he is not in a position to make a rational choice seems foolhardy."' When the medical specialists are divided and uncertain about some aspects of treatment, is it right to just hand over the decision making to the patient, expecting her to be able to come to a rational decision at a time of great anxiety?

MICHAEL BAUM KEVIN ZILKHA

King's College Hospital,

London

Cancer London

JOAN HOUGHTON

1 Baum M. Breast cancer trials-a new initiative. Br Med $\mathcal{F}$ $1981 ; 281: 563-4$

2 Baum M, Houghton J. Current and future management of early breast cancer. Attitude survey amongst participants in CRC trials. Lancet $1980 ; i \cdot 929$.

3 Blamey RW. Adjuvant chemotherapy in breast cancer-the case against. In: Delaney WP, Varco RL, eds. Controversies in against. In: Delaney WP, Varco RL,

4 Early Breast Cancer Trialists Collaborative Group. Effects of adjuvant tamoxifen and cytotoxic therapy on mortality in early
breast cancer: an overview of 61 randomised trials among breast cancer: an overview of 61 randomised
28896 women. N Engl f Med 1988;319:1681-92.

5 Houghton J, Baum M, Nissen-Meyer R. Is there a role for perioperative adjuvant therapy in the treatment of early breas cancer? Eur $\mathcal{F}$ Surg Oncol 1988; 14:227-33

6 Anonymous. Informing patients about clinical disagreement. Lancet 1989;ii:367-8.

\section{Ignorance about listeria}

SIR,-In a recent editorial Dr P J Wilkinson was quite outspoken in denouncing the substantial ignorance about listeria and the lack of a sound basis for translating the flourishing "listeria hysteria" into rational measures for the control of listeriosis. 'An open question, in particular, is the origin of sporadic cases of listeriosis, which with very few exceptions ${ }^{23}$ have not been linked with the consumption of any particular food.

We have recently had the opportunity to report a case of sporadic foodborne listeriosis in a 54 year old woman admitted to intensive care with clinical symptoms of meningitis. Listeria monocytogenes serotype 4 was cultured from her cerebrospinal fluid, and treatment with ampicillin plus gentamicin led to complete recovery within two weeks. With the help of her relatives we examined the remainder of some foodstuffs eaten by her before the onset of her illness which had been kept refrigerated. Two strains of $L$ monocytogenes were isolated, both of serotype 4 , one from a sample of sausagemeat and the other from a sample of fish.

At the Pasteur Institute the clinical isolate and the fish isolate were found to share the same phage pattern (340/108/2389/3552/47/52/107/1444/312) whereas the meat isolate was susceptible to the same first seven phages but was not lysed by others, including phages 1444 and 312. At the same time we examined the three isolates by restriction endonuclease analysis of chromosomal DNA, a technique that has already been used in our laboratory to confirm a case of hospital cross infection with phage untypable $L$ monocytogenes. ${ }^{+}$ With three different endonucleases (BamHI, EcoRI, and HindIIII) the clinical isolate showed DNA fingerprints identical with those of the fish isolate but different from those of the meat isolate. Neither the fingerprints shared by the clinical and the fish isolates nor those exhibited by the meat isolate were identical with any of the $17 \mathrm{~L}$ monocytogenes isolates tested in our laboratory during the past two years.

Both phage typing and DNA fingerprinting confirmed the foodborne origin of this sporadic case of $L$ monocytogenes meningitis and linked it with the consumption of fish (which is an unusual source of foodborne listeriosis), excluding the strain isolated from meat as the cause. Most probably the survival and transmission of the organism was due to undercooking, as the fish had been eaten and then stored in the refrigerator after having been steamed. Moreover, these data suggest a maximum incubation period of three to four days, in agreement with other findings, ${ }^{2}$ but in contrast with a time of one to several weeks estimated by a WHO working group.

Given the inadequacy of serotyping and the high proportion of phage untypable strains, ${ }^{6}$ public health reference laboratories should assume the task of routinely applying modern typing techniques, such as DNA fingerprinting, analysis with DNA probes, ${ }^{7}$ or isoenzyme analysis, ${ }^{8}$ to understand more about transmission of listeria and improve the control of human listeriosis.

We thank Dr Jocelyne Rocourt, Pasteur Institute, Paris, for phage typing.

B FACINELLI

Institute of Microbiology,

University of Ancona Medical School,

-60131 Ancona,

Italy

Institute of Microbiology,

University of Padua Medical School,

I-35121 Padua,

Italy

P E VARALDO

Institute of Microbiology,

University of Modena Medical School

I-41100 Modena,

Italy

Wilkinson PJ. Ignorance about listeria. Br Med $\mathcal{f}$ 1989;299: 276-7. (29 July.)

Kerr KG, Dealler SF, Lacey RW. Materno-fetal listeriosis from cook-chill and refrigerated food. Lancet 1988;ii:1133.

Centers for Disease Control. Listeriosis associated with consumption of turkey franks. MMWR 1989;38:267-8.

Facinelli B, Varaldo PE, Casolari C, Fabio U. Cross-infection with Listeria monocytogenes confirmed by DNA fingerprinting. Lancet 1988;ii: 1247-8.

5 WHO Working Group. Foodborne listeriosis. Bull WHO 1988 ; 66:421-8.

6 McLauchlin J. Listeria monocytogenes, recent advances in the taxonomy and epidemiology of listeriosis in humans. $\mathcal{F}$ App Bacteriol 1987;63:1-11.

7 McLauchlin J, Saunders NA, Ridley AM, Taylor AG. Listeriosis and food-borne transmission. Lancet 1988;i:177-8.

8 Bibb WF, Schwartz B, Gellin BG, Plikaytis BD, Weaver RE. Analysis of Listeria monocytogenes by multilocus enzym electrophoresis and application of the method to epidemiologic investigations. International fournal of Food Microbiology 1989 8:233-9.

\section{Don't put your daughter into medicine}

SIR,-Dr Mary Mather's personal view' has prompted me to give my own. Three of my five daughters have chosen medicine as a career; two of them are working full time in their chosen specialties of hepatology and radiology, and both have two children under 5; the third has recently completed a punishing year as a houseman.

Three factors made possible my own career in medicine, during which I have always worked ful time and have brought up a large family. Firstly, a supportive husband; mine is an academic in medicine, but the profession is immaterial as long as he is keen for you to have a full time career. Secondly, consistent home support, in my case a resident mother in law. I was lucky: my daughters pay much of their salary to someone else to help with their children. Finally, living close to work and being able to slip home for short spells. I enjoyed being at home with my young children but never wanted to be there all the time. I have been a better mother and a much appreciated one as a result.

Criticisms of a career in medicine for women include rigidity of jobs and employers, limited availability of mainstream part time jobs, and difficulty in returning to work after a break in service; all this is true to some extent. I was in grant funded research for 10 years and could have worked part time. There are many such options and part time training schemes exist. The entrenched attitude of some colleagues should not deter you. My view is that once you organise your 